\title{
Telegenetics in Maine: Successful clinical and educational service delivery model developed from a 3-year pilot project
}

Dale Halsey Lea, MPH, RN $N^{1}$, Judith L. Johnson, PhD ${ }^{3}$, Sara Ellingwood, $M S^{1}$, Walter Allan, $M D^{2}$, Arvind Patel, $M D^{4}$, and Rosemarie Smith, $M D^{1}$

\begin{abstract}
Purpose: The goal of this 3-year pilot project was to increase accessibility to genetics educational and clinical services in Maine. Methods: Southern Maine Genetics Services, Foundation for Blood Research in collaboration with Maine Telemedicine Services established telemedicine capacity to link with rural health care centers located in Northern, Central, and Southern Maine and public health nursing statewide for the provision of genetics clinical and educational services. Core partners included a rural family practice residency program, a rural pediatric practice in northern Maine, and public health nurses statewide. The telegenetics model created was based on development and implementation of a preventive and medical management technology solution, conducting a pilot study to collect data, and approaching insurance companies for reimbursement. Evaluation included surveys on the quality, acceptability, and usefulness of genetics services delivered via telemedicine, telephone interviews, and decision-making confidence evaluations. Results: During the project period, 24 rural clinical sites participated. In total, 93 presentations were given, and 125 patients were evaluated. Sixty-four percent of patients evaluated were pediatric. Despite site coordinator efforts to complete satisfaction surveys, the provider and patient response level was low ( $18 \%$ and $25 \%$, respectively). Of those evaluations received, provider and patient response to telegenetics was positive. Decision-making confidence for genetics and neurology consultants was high. Our experience contributes to the development of telegenetics models that can be used in other rural states. Genet Med 2005:
\end{abstract} 7(1):21-27.

Key Words: telemedicine, telehealth, rural, clinical genetics, genetics education

Individuals and families living in rural areas of the United States face significant barriers that prevent access to health care including genetics services. ${ }^{1,2}$ Maine is among those rural states, having a large geographic area and a small population of slightly more than a million people living in many scattered townships, island communities, and plantations. Many rural, isolated, and underserved populations in Maine are challenged to obtain quality health care due to poverty, inadequate health insurance coverage, limited availability of public transport, prolonged periods of hazardous winter driving conditions, and poor road infrastructure. There is restricted availability and maldistribution of specialty health care providers, including genetics specialists, which are mainly concentrated in the South-Central part of the state. At present, there are four med-

From ${ }^{1}$ Southern Maine Genetics Services and ${ }^{2}$ Clinical Services, Foundation for Blood Research, Scarborough, Maine; ${ }^{3}$ Office of Institutional Research, University of Southern Maine; and ${ }^{4}$ Maine Telemedicine Services, HealthWays/Regional Medical Center at Lubec, Maine. Dale Halsey Lea, MPH, RN, Assistant Director, Southern Maine Genetics Services, Foundation for Blood Research, 69 U.S. Route \#1, PO Box 190, Scarborough, ME 04070.

Received: July 23, 2004.

Accepted: September 1, 2004.

DOI: 10.1097/01.GIM.0000151150.20570.E7 ical geneticists in Maine with three in the South, and one in the Northern part of the state. Health care providers and patients, therefore, do not have equity of access to genetics services.

Broadly defined, telemedicine is the transfer of electronic medical data (i.e., high-resolution images, sounds, and patient records) from one location to another and includes live synchronous interactive televideo sessions. ${ }^{3}$ Interactive telemedicine technology-based services are an appealing health care delivery model for large areas of rural Maine and have been successfully used in Georgia, a rural state with similar access to services issues for children with special health care needs, including genetic conditions. ${ }^{4}$ Telehealth services for the provision of cancer genetic counseling have also been successfully piloted in the United Kingdom and in Australia.,5 The American Academy of Pediatrics, in their 2004 Technical Report, notes that telemedicine's strengths-visual diagnosis and counseling - have an obvious application to genetics and dysmorphology, and application of this technology should be "well suited for effectiveness studies." (p. 641) ${ }^{7}$

This article describes the 3-year pilot outreach experience to provide clinical and educational genetics services via telemedicine, and the data collected from October 1, 2000 through September 30, 2003. The pilot project, InTeleGen: Outreach Genet- 
ics Clinical and Educational Pilot Project, was funded by the National March of Dimes Mission Investment Opportunity Program. Clinical and educational outreach activities targeting physician professional associations and grass roots education for providers and Maine communities are described.

\section{MATERIALS AND METHODS}

The Maine Telehealth Network (MTN), a project of HealthWays/Regional Medical Center at Lubec, Maine, was instituted to increase access to health care, mental health, social services, and distance education throughout Maine using synchronous televideoconferencing (ITV) technology over Integrated Services Digital Network (ISDN) phone lines. ${ }^{8}$ This network has developed through a series of federal and foundation grants beginning in 1997. In the fall of 2000, MTN established ITVbased telemedicine capacity at Southern Maine Genetics Services, Foundation for Blood Research (SMGS/FBR) with a Polycom 512 Viewstation, 32-inch monitor, rolling cart, and associated cabling and supplies. The ITV equipment, at each end of the communication process, consists of a television, a small programmable unit with attached video camera, and microphone. There is a large range of available add-on equipment, such as handheld cameras, otoscopes, electronic stethoscopes, and document cameras. The cameras on the ITV are controllable at either end by the use of a remote control, very similar to and as easy to operate as the common television remote. SMGS/FBR installed a multipoint ITV unit to facilitate simultaneous linkage with two or three other ITV units at the same time, to enable case management and educational programs to multiple sites. Genetics clinical examinations were conducted using 3 ISDN lines (384 kbps) for better picture resolution. The peripheral equipment most often used was a printer for patient photography.

The pilot project was a collaborative effort between SMGS/ FBR and three core health agency partners, representative of rural Maine communities. Maine public health nursing, consisting of 60 nurses statewide, referred and/or accompanied patients and families to telemedicine genetics consultations. Maine Dartmouth Family Practice Residency Program has 27 residents located in a rural host institute and four other rural medical centers. The residents participated in genetics education and clinical consultations over ITV. Cary Medical Center, 300 miles north of SMGS/FBR, served as a primary outreach clinical and education site for the project, providing services for a large, rural geographic area.

Patients for telegenetics clinical consultations were scheduled by the three participating health agency's site coordinators in consultation with the SMGS/FBR site coordinator. In addition, patients in need of neurology evaluation were scheduled, because SMGS/FBR has a pediatric neurologist on staff. Some of the neurology evaluations were combined with genetics evaluations or lead to a follow-up telegenetics evaluation. The outreach site coordinator provided the patient with an orientation to telemedicine, and family and medical history forms to complete before the consultation. When the patient arrived at the outreach site, the nurse reviewed the procedures for the telemedicine consultation, patient confidentiality, record keeping, and the informed consent form for participation in the telegenetics consultation. The signed informed consent form was completed and sent by fax to SMGS/FBR before the consultation. As a part of the encounter, the outreach site nurse performed necessary physical measurements. The practitioner or outreach site nurse also provided follow-up health teaching, facilitated completion of medical testing, and coordinated follow-up procedures as needed.

After the initial telegenetics consultation and relevant tests were completed, SMGS staff, the primary care provider, and the patient determined whether a follow-up visit in person was needed. These patients were either seen at SMGS/FBR or at an outreach site-visit, held once a year for participating sites.

Information collected on each telegenetics consultation included the type of patient (preconception/prenatal, pediatric, or adult) and the presenting problem. Throughout the project period, a subset of information was collected from participating patients, families, and providers, using a Patient and Provider Satisfaction Questionnaire developed by MTN. Educational presentations were also evaluated. Telephone interviews were conducted in year 3 of the project. The research team selected patients and providers representative of the different types of patients (e.g., prenatal, pediatric, adult, or cancer), the various types of primary care providers (e.g., family practitioners, physician assistants, or nurses), and participating sites (e.g., rural hospital or rural health center). The purpose of the interviews was to obtain detailed qualitative data about acceptability of and satisfaction with telegenetics services. A retrospective analysis of consultant decision-making confidence was also performed. For this analysis, decision-making confidence and satisfaction were measured using an investigatormodified decision confidence scale originally developed by Sanders and Courtney, ${ }^{9}$ and later adapted by Brennan et al. ${ }^{10}$ and Young and Ireson. ${ }^{11}$

\section{RESULTS}

\section{Establishing our network}

In the first year, SMGS established an Advisory Board whose members included representatives from the core project sites, March of Dimes, and a telehealth consultant from Georgia who had extensive experience with building telehealth capacity for genetics and related services. SMGS/FBR also conducted a needs assessment with physicians, residents, and nurses from the three core clinical sites to introduce the concept of telemedicine use for genetics services, determine practitioners' familiarity with telemedicine, and to identify genetics topics of interest for educational presentations. Advantages of using telemedicine for genetics services cited by respondents included (1) increased access to genetics and experts, (2) convenience, and (3) decreased travel time. Disadvantages cited were (1) potential problems with the equipment, (2) a less personalized approach, and (3) lack of in-person interaction between the patient and doctor. Thirty-nine percent of respondents 
said that they would like to receive genetics continuing education, clinical evaluation, and consultation services using telemedicine about 4 times a year. Thirty-five percent of the practitioners surveyed said they had patients who they would consider appropriate for telemedicine consultation. Following the needs assessment, site visits were conducted using ITV.

\section{Barriers to establishing the telegenetics network}

During Year 1, four barriers to provision of genetics services by telemedicine were identified. The first was lack of knowledge about what telemedicine services are and how they are used. This was reflected in the needs assessment surveys. A second barrier was the lack of understanding of the role current genetic services could play in the care of patients, both by the providers and the patients themselves. The location of the ITV unit was another unforeseen barrier. In some health care organizations, the ITV unit is in the emergency room, making it difficult for patients to be referred for services. In some organizations, the ITV was in a conference room with a set schedule for administrative and educational programming. The fourth barrier relates to hospital credentialing and privileging processes for the SMGS physicians. Each hospital has its own specific requirements and a process that in general is lengthy and requires substantial documentation and an application fee. These barriers were addressed throughout the project period.

\section{Attempts to improve utilization of telegenetics}

During the second and third years, SMGS/FBR used two approaches to improve providers' use of telemedicine for genetics services to increase access to genetics services for Maine residents, and the ability of health care providers to identify appropriate genetics referrals. The first approach targeted physician associations. Presentations on the telegenetics activities were made to various physician associations statewide. In addition, the Executive Director of MTN sits on the Maine Medical Association (MMA) Public Health Committee. In collaboration with the Executive Director, SMGS/FBR drafted a resolution regarding the importance of physician education in genetics that was presented by the MMA Public Health Committee to MMA members recommending that MMA collaborate with other professions and groups to establish standard protocols for genetic referral. This resolution was successfully passed at the annual meeting of the MMA membership in the spring of 2003. The second approach targeted physician practices and other providers individually and within their own settings, offering them on-demand clinical and educational genetics services, newsletters, and tailored telegenetics pamphlets. For example, a news sheet The Penguin was created and sent to all Maine providers to inform them of the availability of telegenetics clinical and educational services and reasons for referral. SMGS also worked with MTN to introduce telegenetics to interested pilot sites.

The genetics educational component combined requested genetics educational programs and clinical consultations. The genetics educational programs were presented to health service providers by ITV to their own remote practice setting with Continuing Medical Education (CME) and Continuing Education Unit (CEU) credits awarded. Topics were selected based on practical interest of targeted groups, with scheduling on demand. A case-based educational approach was used, and for clinical consultations, practitioners were encouraged to present their own patients to genetics specialists and take part in the consultation, follow-up, and management. When a practitioner was not available, the outreach site nurse presented the case and facilitated the telemedicine encounter.

\section{Summary of 2-year telegenetics network}

Far exceeding projected numbers, SMGS/FBR, in collaboration with Maine Telemedicine Services (MTS), linked to 24 sites (Fig. 1) selected from the Maine Telehealth Network which, as of May 2004, consists of over 250 interactive televideoconferencing (ITV) sites among 110 health, social, and mental health provider organizations. Sites linked to SMGS/FBR to date include 13 hospitals/medical centers, eight small, rural health clinics, two private practice offices, and one community action program for women and children.

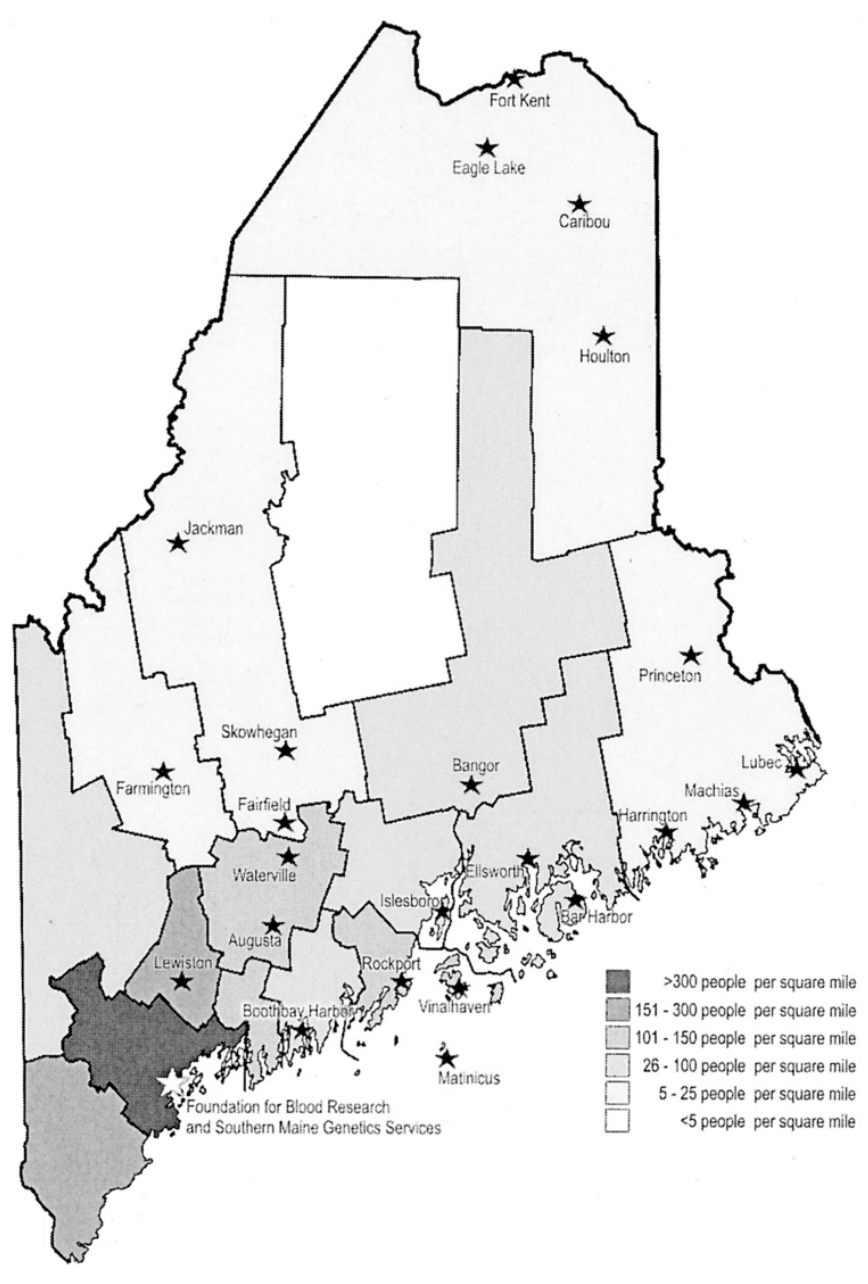

Fig. 1. SMGS telemedicine sites. 


\section{Educational programs}

Educational programs were interdisciplinary, involving physicians, physician assistants, nurse practitioners, nurses, and the public. A total of 93 genetics and related educational programs were presented during the 3-year grant period to more than 650 participants. During Year 1, 14 presentations were given. By Year 2, the number of presentations grew to 35 and by Year 3, the number given was 44 . Genetics presentations given to providers and the local communities were also provided to three island communities. Topics covered prenatal, pediatric, and adult-onset genetic conditions and issues.

Evaluation tools for presentations were designed by participating institutions conferring CMEs and CEUs. Because the evaluation tools were developed by each individual institution, they differed somewhat in their structure and evaluation scale. Most evaluation tools used a 4-point Likert scale, rating presentations as poor, fair, good, or excellent. Evaluations from $85 \%$ of the presentations given during the project period were received and analyzed. Overall, evaluations were very positive and comments reflected participants' excitement and receptiveness to the genetics information that was delivered. The mean score for all presentations was 3.88 on a 4-point scale (4 being excellent). Participants commented that the "interaction between the speaker and staff was great." The few difficulties noted were technical and included problems connecting to a distant site, having the connection interrupted, and not being able to read densely worded slides.

\section{Genetic consultations}

From March 1, 2001 through September 30, 2003, the number of patients and families evaluated increased from 17 during the first year to 50 in the third year with composite total of 105 patients and approximately 250 family members. By the end of the third year, telegenetics patients made up $13 \%$ of all patients seen by SMGS. The majority of patients seen were pediatric $(64 \%)$, with the most common reason for referral being to rule out an underlying genetic syndrome as a cause of developmental delay. This percent is higher than the number of pediatric patients evaluated in person at SMGS; during the project period, $44 \%$ of patients were pediatric. This difference can perhaps be explained by the fact that the core site, Cary Medical Center, Pines Pediatrics, referred the most patients thus skewing the numbers of pediatric patients evaluated. In addition, this site was the most distant and providers were more familiar with SMGS staff than other sites. Table 1 summarizes the types and numbers of patients evaluated during the project period.

Follow-up consultation was recommended in 33 patients. One patient was referred to the metabolic clinic for follow-up. Of the remaining 32 patients requiring follow-up, 6 patients were evaluated in person as recommended to pursue diagnosis or because of patient/family preference, representing $6 \%$ of the total number of patients. With these patients, geneticists had the opportunity to conduct the physical examination in person to assess the accuracy of the dysmorphology evaluation conducted via telemedicine. In one case, a diagnosis of Kabuki
Table 1

Type and number of patients evaluated and counseled by telemedicine at SMGS/FBR during the project period

\begin{tabular}{lrr}
\hline Patient type & $N$ & $\%$ \\
\hline Pediatrics & 32 & 30 \\
Genetics only & 27 & 26 \\
Neurogenetics & 8 & 8 \\
Neurology only & & \\
Adult & 15 & 14 \\
$\quad$ Cancer & 16 & 15 \\
Other & 3 & 3 \\
Reproductive genetics & 4 & 4 \\
Prenatal & 105 & 100 \\
$\quad$ Preconception & & \\
Totals & & \\
\hline
\end{tabular}

syndrome was made in a pediatric patient from photographs taken during the telemedicine dysmorphology examination. Follow-up in-person examination confirmed the diagnosis. For the other 26 patients requiring follow-up, telemedicine was judged a satisfactory means of follow-up by the SMGS staff, primary care provider, and patient.

\section{Evaluation of telegenetics clinical services}

Satisfaction Evaluation Questionnaires were used to evaluate clinical telemedicine sessions in which primary care providers presented their patients; one instrument evaluates providers' response to the telemedicine, visit, and the other elicits patient responses. We enlisted the nurse site coordinators to facilitate patient and provider evaluation responses. Even with their assistance, only $18 \%$ of provider participants returned Satisfaction Evaluation Questionnaires. Of those satisfaction surveys received, provider satisfaction with all sessions was rated with a mean of 3.83 on a 4-point scale with 4.0 being very satisfied and 1.0 being very dissatisfied. Sessions were virtually problem-free. Minor difficulties reported included interrupted connection and human error operating the equipment. These problems were regarded as having only minimal effect. Satisfaction was high with the quality of technical support provided by MTN.

During the project period, a Patient Satisfaction Evaluation Form was also provided to all patients after a telegenetics consultation. As with provider evaluations, even with assistance of the site coordinators, only $25 \%$ of participating patients returned the evaluation form. Patients who responded were very satisfied with the care they received during the sessions with an overall mean rating of 3.56 on a 4-point scale, with 4.0 being very satisfied and 1.0 being very dissatisfied. When indicating the best thing about using telemedicine for care, all reported convenience in terms of travel. Other positive factors noted were that family members could be involved in the sessions, and that all professionals involved in providing health care 
(referring provider and specialist) were included as well. With respect to what they did not like about using telemedicine for care, two said that they would rather see the doctor in person.

In the spring of 2003, telephone interviews were conducted with patients and providers, selected by the research team as representative of the different types of patients, providers, and sites as part of Year 3 evaluation. A total of 10 patients and 11 providers were contacted by mail to ask for their participation. Interviews were conducted with six patients and eight providers.

The six patient interviewees learned about telemedicine through a variety of sources including contact with a participating site. All had used telemedicine only once by the time the interviews were conducted. Most had used it for genetic counseling or to get a second opinion. All patients said they were "very comfortable" with their interaction with SMGS/FBR staff and all rated telemedicine as "very personal." All felt that their consultation was successful and addressed their problem correctly. Patients cited savings of time and travel, and convenient access to first-rate medical care without having to leave their communities as benefits of telemedicine. With respect to drawbacks, three said there were none, one cited voice delay as a drawback, and one mentioned lack of hands-on examination, which in their case was not critical.

Most of the eight providers had learned about telemedicine through some aspect of their job. All providers said they felt that their patients' problems were understood and correctly addressed. With respect to benefits of telemedicine, providers cited access to specialists, travel savings, and the immediacy of the response. Absence of personal touch/hands-on examination was cited as a drawback. Some providers also said that there were some technical problems such as inability to connect with SMGS and interrupted or dropped connection.

Results of decision confidence of telemedicine consultants are presented in Table 2. The consulting geneticist and pediatric neurologist responded to a 6-item Likert-type scale ranging from strongly disagree to strongly agree. The mean response for each item was determined for the decision confidence scale. Overall, the consulting geneticist and neurologist felt confident in evaluating and managing patients seen via telemedicine.

\section{Table 2}

Decision confidence of telemedicine consultants $(N=105)$

\begin{tabular}{lc}
\hline Statement & Rating \\
\hline $\begin{array}{l}\text { I had access to as much of this patient's pertinent medical record as I } \\
\text { would for an in-person visit. }\end{array}$ & 5.61 \\
$\begin{array}{l}\text { Using the telemedicine system, I was able to elicit a good history of } \\
\text { the patient's medical condition. }\end{array}$ & 5.81 \\
I was able to identify the real concern(s) of this patient/family. & 5.81 \\
I was able to get pertinent physical exam information. & 5.33 \\
$\begin{array}{l}\text { I felt confident in treating this patient's problems using telemedicine } \\
\text { equipment. }\end{array}$ & 5.54
\end{tabular}

The telemedicine equipment worked well today. 5.29

Ratings were on a scale of $1-6$ with 6 being strongly agree.

\section{Cost and benefits of telegenetics}

One of the most satisfactory components of telegenetics for patients and families was the convenience from reduced travel. As an example, one of the core participating sites, Cary Medical Center is located in Caribou, 608 miles round-trip from SMGS. Patients evaluated by telemedicine appreciated a savings both in time and in reduced travel. In Maine, the official cost of travel is $\$ 0.32 / \mathrm{mile}$. Therefore a round trip from Cary Medical Center, Caribou to the SMGS would cost $\$ 194.56$ for travel. When lodging for one night and food for two days is included the cost to a family would be approximately $\$ 350$. This does not include the time lost from work for one or both parents. When the SMGS interdisciplinary team travels to Cary Medical Center, the cost of their travel time (nonbillable hours) would also be included. The cost of ISDN lines and consultation using telemedicine is $\$ 240 /$ month. This cost was covered during the grant period, and is currently being paid for by state genetics grant funding.

\section{DISCUSSION}

Provision of genetics services in any largely rural state like Maine, with a limited number of genetics specialists, is challenging for providers and patients because of the cost, time, logistics, and stress associated with long distance travel, often in hazardous winter driving conditions. The scarcity of genetics service providers is a deterrent for some providers to refer patients for genetics consultation. Telemedicine offers an accessible and acceptable venue for meeting the growing need for genetics education for primary care providers and clinical genetics services for individuals and families separated from genetics specialists by distance. Current technology used by SMGS/FBR allows geneticists to provide relevant, on-demand genetics educational presentations to primary care providers. The technology also allows specialists and patients to see and talk to each other in real time. As documented in other studies, ease of use, quality of transmission, and convenience influence provider acceptance. ${ }^{10}$ By moving information rather than the patient, telemedicine promises to enhance health care while dismantling the barrier of where and when genetics services are provided.

We found telemedicine to be equivalent to in-person genetics consultations, and useful for conducting many aspects of genetic evaluation and counseling. Specifically, telemedicine is useful for obtaining and reviewing family and medical histories, for physical examination and dysmorphology evaluations, and for prenatal and cancer genetic counseling. These aspects are easily accomplished using telemedicine. Genetic evaluations conducted via telemedicine for patients at risk for metabolic or neurocutaneous disorders do not lend themselves to telemedicine evaluation because the genetics specialist cannot palpate organs or view the skin with a Woods lamp.

Our experience with telemedicine demonstrates the importance of building on a telemedicine infrastructure to develop a telegenetics program. The ability to collaborate with the estab- 
lished Maine Telehealth Network (MTN), with its operational expertise and technical support infrastructure were major factors in SMGS/FBR's ability to expand clinical and education genetics services to rural areas. The Maine telemedicine system is a consortium of networks. In the United States and other parts of the world, this type of network has not yet proven to be self-sustaining and well coordinated. ${ }^{8,12}$ Furthermore, the literature reveals that there has been little research specifically focused on assessing program success based on sustainability. ${ }^{7}$

In Maine, sustainability of the telemedicine network relies on collaborative partnerships with participating sites through a written agreement that includes graded increases in membership fee and federal grant support. Grant funds were largely used to purchase equipment for rural access facilities and to subsidize other MTN efforts to develop services. Network fees from a large number of member organizations (over 250) plays a part in sustaining the professional network staff base required for the integrated telemedicine services provided by MTN. However, grants and services contracts for collaborative contracts remain the major funding support for the network. ${ }^{8}$

Sustainability for SMGS continued delivery of genetics services via telemedicine relies on reimbursement from payers for clinical genetics services. SMGS has achieved Maine Medicaid reimbursement for clinical genetics evaluations. Sustainability is also achieved by cost savings on travel to remote sites to provide genetics services. However, these sources of income do not completely cover the costs of the ISDN lines, telephone dial up costs, and network fees. A state genetics grant and institutional contributions also fund SMGS telegenetics services.

In developing the telegenetics model, we encountered four main barriers to provision of genetic services by telemedicine. We addressed the first barrier-lack of knowledge about what telemedicine services are and how they are used-in two ways. SMGS worked with MTN to introduce telegenetics to interested pilot sites. We also created a news sheet that was sent to all Maine providers to inform them of the availability of telegenetics and reasons for referral. The second barrier-lack of understanding of the role current genetic services could play in the care of patients - was addressed by providing genetics education sessions to both providers and community members. These presentations have served to increase awareness of and interest in genetics as evidenced by increased referrals. The third barrier-location of the ITV unit— has been difficult to overcome. This is because some institutions such as hospitals have funded telemedicine for emergency room and administrative use only, making it difficult for patients to be referred for services. We have been able to work with some hospitals to increase use of the conference room telemedicine units for prenatal and cancer counseling. We are currently working with MTN to address the fourth barrier-hospital credentialing and privileging processes for the SMGS physicians. MTN is actively seeking a means for uniform credentialing and privileging that would allow transfer of credentialing privileges from one hospital to another. Once accomplished, this would ultimately shorten the application process and reduce the costs of application fees.
As we have addressed these barriers, telegenetics in Maine has increasingly worked well for delivery of needed services. The patients have received "face-to-face" counseling over ITV. Extended family members have been able to participate in consultations. Many of the pediatric genetic evaluations did not require direct physical patient contact, as much of the clinical examination was based on evaluation of observable physical features. Genetics specialists, using ITV, obtained appropriate measurements with the aid of a trained clinic nurse at the distant site and were able to assess developmental level and quality and nature of movements. The remote control of the camera made it possible to "zoom in" on particular features of interest and take still photos for documentation. The strengths of telemedicine, visual diagnosis and counseling, as described in the American Academy of Pediatrics 2004 Technical Report, ${ }^{7}$ made easy application to provision of genetics clinical services possible. The high scores reported on the decision-confidence scale indicate that the consultants were confident in using telemedicine for genetics and neurology evaluations and satisfied with this clinical format.

In our initial provider needs assessment, providers did not specify whether "appropriate" referrals applied to patients with visible symptoms that would lend themselves to a visual diagnosis using telemedicine technology, or whether appropriate patients were those for whom finances and distance were barriers to receiving in-person evaluation. Further research using focus groups and interviews would strengthen this assessment.

The project evaluation using satisfaction questionnaires was not as robust as we hoped. Therefore, it was not possible to draw strong conclusions about the acceptability and usefulness of telegenetics. The limited response that we received and the result of the telephone interviews indicated that telemedicine may effectively approximate many aspects of an in-person exam of patients by providers, and can save much time and effort for travel by rural patients or providers.

Further studies that would help to clarify the level of patient provider satisfaction and provider referral information should be considered.

In sparse, dispersed population groups, such as in Maine, adequate system utilization can only really be achieved by a statewide approach to service delivery, to multiple sites, from agencies such as the public health genetics program. This model enables economies of scale, concentration of the limited pool of expertise in telegenetics to develop clinically effective telemedicine services, and an infrastructure of consistent policies and advocacy on such issues as informed consent process and revenue streams conducive to sustainability. In 2004, the Maine State Technical Advisory Council for Genetics was established. One of the tasks of this genetics provider-based group is to develop a strategic plan for a comprehensive, statewide program for genetic services in cooperation with the Maine Bureau of Health, including further evaluation of telemedicine as a means to achieve statewide genetics services.

The SMGS/FBR telegenetics model based on the development and implementation of a preventive and medical man- 
agement technology solution, conducting a pilot study to gather data, and approaching insurance companies for reimbursement with gathered data has shown that delivery of genetics services using ITV can be successful. New program development will require grants and a business planning process for sustainability with consideration of avoided costs and enhanced access to services and patient retention.

The continued involvement of physician and provider associations is strategically important to mainstreaming telegenetics and the development and implementation of best practices for genetics. Our experience indicates that telehealth approaches in Maine are enabling equity of access to services for rural, isolated, and underserved populations and introducing efficiency for the delivery of genetics educational and clinical services. The strategies and lessons learned and the model for the delivery of genetics services in Maine may have application in other states.

The future holds great promise for genetics. Combined with interactive telemedicine technology, the future is even brighter for the population we serve.

\section{ACKNOWLEDGMENTS}

The authors gratefully acknowledge the National March of Dimes Mission Investment Opportunity Program (MIOP) for funding our 3-year pilot project, and Sharon Schulberger, Director of Program Services, March of Dimes, Maine Chapter for collaboration in our project.

\section{References}

1. Guttmacher AE, Jenkins J, Uhlman WR. Genomic medicine: Who will practice it? A call to open arms. Am J Med Genet (Semin Med Genet) 2001;106:216-222.

2. Ricketts TC. The changing nature of rural health care. Annu Rev Public Health 2000;21:639-657.

3. Telemedicine Information Exchange, 2003. Available at: http://tie.telemed.org.

4. Karp WB, Grigsby KR, McSwiggan-Hardin M, Pursely-Crotteau S, Adams LN, Bell $\mathrm{W}$ et al. Use of telemedicine for children with special health care needs. Pediatrics 2000; $105: 843-847$

5. Gattas MR, MacMillan JC, Meinecke I, Loane M, Wootton R. Telemedicine and clinical genetics: establishing a successful service. J Telemed Telecare 2001;7(suppl 2):68-70.

6. Gray J, Brain K, Iredale R, Alderman J, France E, Hughes H. A pilot study of telegenetics: Letter to the editor. J Telemed Telecare 2000;6:245-247.

7. American Academy of Pediatrics, Technical Report. Telemedicine: Pediatric applications. PEDIATRICS 2004;113:639-643.

8. Edwards MA, Patel AC. Telemedicine in the state of Maine: A model for growth driven by rural needs. Telemed J E Health 2003;9:25-39.

9. Saunders G, Courtney J. A field study of organizational factors influencing DDS success. MIS Quarterly 1985;9:77-88.

10. Brennan PF, Moore SM, Smyth KA. The effects of a special computer network on caregivers of persons with Alzheimer's disease. Nurs Res 1995;14:162-172.

11. Young TL, Ireson C. Effectiveness of school-based telehelath care in urban and rural elementary schools. Pediatrics 2003;112:1088-1094.

12. Shannon G, Nesbitt T, Bakalar R, Kratochwill E, Kvedar J, Vargas L. Organizational models of telemedicine and regional telemedicine networks. Telemed J E Health 2002;8:61-70. 